$\mathrm{X}-616-68-224$

PREPRINT

\title{
DIRECTIONAL DISCONTINUITIES IN THE \\ INTERPLANETARY MAGNETIC FIELD
}

\author{
Leonard F. Burlaga
}

Laboratory for Space Sciences

June 1968

Extraterrestrial Physics Branch Preprint Series

GODDARD SPACE FLIGHT CENTER

Greenbelt, Maryland 
PRECEDING PAGE SLANK NOT FILMED.

\title{
DIRECTIONAL DISCONTINUITIES IN THE INT ERPLANETARY MAGNETIC FIELD
}

Leonard F. Burlaga ${ }^{1}$

\begin{abstract}
It is shown that the interplanetary magnetic field has different characteristics on different scales, and it is noted that a given physical theory may not be applicable or relevant on all scales. Four scales are defined in terms of time intervals on which the data may be viewed. Many discontinuities in the magnetic field direction are seen on the mesoscale $(\approx 4$ days, $\approx 1 \mathrm{AU})$. The characteristics of such directional discontinuities which were observed by Pioneer 6 during the period December 16,1965-January 4,1966 are presented, with special emphasis on their distribution in time. Previously, it was suggested that such discontinuities are simply boundaries of spaghetti-like filaments extending from the sun to the earth. Here it is shown that on the mesoscale unique filaments with sharp boundaries containing well-ordered magnetic fields are not always seen although discontinuities are always present at $\approx 1 \mathrm{AU}$. Thus, the interplanetary medium appears to be discontinuous rather than filamentary. The filamentary model implies that discontinuities originate at the sun and are convected with the solar wind. The discontinuous model allows the additional possibility that the discontinuities form in the interplanetary medium far from the sun.
\end{abstract}

$\widetilde{1_{\text {NAS-NRC Resident Research Associate }}}$ 


\section{INTRODUCTION}

The existence of numerous discontinuities in the magnitude and the direction of the interplanetary magnetic field was established by Ness et al. (1966). The existence of marked and abrupt changes in the direction of anisotropy of low energy $(\approx 10 \mathrm{MeV})$ solar protons was demonstrated by Bartley et al. (1966). A strong correlation between the direction of anisotropy of low energy solar protons and the magnetic field direction, shown by McCracken and Ness (1966), supports the inference of Bartley et al. that discontinuities in the direction of anisotropy are due to discontinuities in the magnetic field direction. Observations of numerous discontinuities during the early days of the flight of Pioneer 6 have led all of the above authors to suggest that the interplanetary medium consists of numerous identifiable filaments.

They describe a filament as a cylindrical flux tube which has a diameter $\approx(.5-4) \times 10^{6} \mathrm{~km}$ and which extends from the sun to the earth; boundaries of filaments are assumed to be distinct, and the filaments are assumed to be intertwined and twisted like pieces of spaghetti. The observation of a discontinuity in magnetic field magnitude direction or intensity is said to indicate the passage from one filament to another.

Further observations by Pioneer 6 also show numerous discontinuities, so it appears that discontinuities are a fundamental feature of the interplanetary medium, during this period near solar minimum. However, the existence of an ensemble of filamentary tubes with distinct boundaries enclosing well-ordered 
magnetic field lines is not always clearly demonstrated by these data as we shall show below.

Discontinuities in the direction of the magnetic field are more frequent than discontinuities in the magnitude of the field, so they are of special interest with regard to the structure of the interplanetary medium. Burlaga and Ness (1968) and Burlaga (1968) have studied some characteristics of a small set of particularly clear, isolated directional discontinuities, where a directional discontinuity was defined as a change in the field direction $>30^{\circ}$ which occurs in less than 30 seconds. They found that, 1) the change in the field direction tends to be small, 2) there is a preferred direction associated with the discontinuities, 3) the magnitude of the magnetic field does not change appreciably across most directional discontinuities, and 4) the plasma parameters usually do not change significantly across the directional discontinuities.

In this paper the study of directional discontinuities is continued with special emphasis on their distribution in time. The significance of this distribution with regard to the structure of the interplanetary medium is also discussed. The results are based on Pioneer 6 data which were obtained with a fluxgate magnetometer that is described by Ness et al. (1966). Data for the interval December 16, 1965-January 4, 1966 are presented. During this interval, the spacecraft moved from the earth to a distance $\approx 3 \times 10^{6} \mathrm{~km}$ in a direction normal to the earth-sun line. 


\section{SCALE}

Since Pioneer 6 magnetic field measurements were transmitted at intervals of 1.5 seconds at the highest bit rate and continued for several months, it is possible to look at the data on a variety of scales spanning seven decades in time (10 sec to $\left.10^{7} \mathrm{sec}\right)$. Different characteristics of directional discontinuities are seen on different scales. In this section, the subject of scale is discussed and four useful scales are defined.

Figure 1 shows a large scale view of the interplanetary magnetic field. Hourly averages of the Pioneer 6 magnetic field vector data are plotted for the 20-day interval December 16, 1965-January 4, 1966. The top graph shows the magnetic field intensity and the lower graphs in Figure 1 show the magnetic field direction in solar ecliptic coordinates, $\phi$ being the direction measured in the ecliptic plane with respect to the earth-sun line, and $\theta$ being the direction above $(+)$ or below $(-)$ the ecliptic plane. Certain large scale features are evident in Figure 1: The tendency for the field to be directed in the ecliptic plane; the tendency to be aligned near the spiral direction $\left(\phi \approx 130^{\circ}\right.$ and $\left.\phi \approx 310^{\circ}\right)$; the sector structure $\underset{\sim}{\mathrm{B}}$ directed away from the sun in the intervals December 16December 21 and December 26-January 2, B directed toward the sun in the intervals December 12-December 24 and January 2-January 4) the average intensity of $4 \gamma$. On this scale one might speak of an average field on which fluctuations are superimposed.

On a smaller scale, however, the interplanetary magnetic field looks very different. This is illustrated in Figure 2, which shows $|\mathrm{B}|, \theta$ and $\phi$ for the 24 
hour interval on December 19, 1965. The scale differs from that in Figure 1 by a factor of 10. Numerous discontinuities can be seen in Figure 2. These are the discontinuities which are referred to in the introduction. Particularly notable discontinuities can be seen at 0500,0900 and 1600 UT in Figure 2, but many other abrupt changes are evident. Fluctuations in the field can also be seen. Note that there are larger fluctuations and more discontinuities during the first third of the day than during the last third. This illustrates the general observation that on this scale the interplanetary medium is inhomogeneous and it is difficult to select a given period which is typical. Variability on a scale of a few days is evident in Figure 1. Some "filaments" can be seen in Figure 2; for example, a large filament can be seen in the interval 0300-0600 UT and a smaller filament in the interval between 1500 and 1600 UT. On the other hand, there are intervals when filaments cannot be seen, e.g., 1800-2100 UT.

On a smaller scale of an hour, large discontinuities are seen more clearly, very small discontinuities become visible, and fluctuations are generally less evident. Figure 3 shows three 1-hour intervals from the period in Figure 2. During the interval 2000-2100 UT, the field direction was very uniform, but a small discontinuity can be seen at 2027 UT and a small gradient appears near 2050 UT. During the interval 1500-1600 UT, a large discontinuity can be seen at 1539 UT (the magnetic field direction changes by nearly $90^{\circ}$ ) and a large gradient appears between 1550 UT and 1600 UT. The concepts of turbulence or randomness seem inappropriate for the fields in Figure $2 b$ and $2 c$. The interval 
between $0500 \mathrm{UT}$ and $0600 \mathrm{UT}$ in Figure 2a contains two notable discontinuities and the $\phi$ direction of the field varies through $170^{\circ}$ while the $\theta$ component is constant within $\approx 30^{\circ}$. Discontinuities in the field direction $\left(>30^{\circ}\right.$ in less than 30 sec) can be seen, and appear distinct from the smaller fluctuations and gradients. These are the directional discontinuities which are the subject of this paper. Since Pioneer 6 measures $\underset{\sim}{\mathrm{B}}$ at 1.5 sec intervals, it is possible to view these data on a scale of $\approx 30$ sec. On this scale, most of the directional discontinuities still look discontinuous, but some have a structure which can be observed as shown in Figure 4. Discontinuities on this scale are discussed in an important paper by Siscoe et al. (1968) which is based on Mariner 4 observations during 1964. In general, the term discontinuity is inappropriate on this scale, so Siscoe et al. have introduced the term "current sheet" because it is a current sheet which maintains the field change. An analysis of wide ( $\gg 10 \mathrm{sec})$ current sheets showed that the field tends to change directions by rotating in a plane as one would expect for a hydromagnetic tangential discontinuity (Colburn and Sonett, 1966). This is the situation shown in Figure 4. Such rotation fans are relatively rare, however. The thickness distribution observed by Siscoe et al., shows that roughly $50 \%$ of the transitions in the duration range $0-5$ minutes had durations $<14 \mathrm{sec}$, and $10 \%$ with durations $<5$ minutes had durations $<2$ seconds. Thus, on a scale of one hour, current sheets can be considered to have zero thickness and directional discontinuities can be considered to be true discontinuities in the sense of hydromagnetic theory. 
The above discussion of Figures 1-4 shows that different structures can be seen in the interplanetary medium when it is viewed on different scales. The difference is not just one of appearance, for different theories must be applied on the various scales. Parker (1963) has shown that an aerodynamic theory will explain the very large scale features of the interplanetary medium. Burlaga (1968) has shown that magnetohydrodynamics is applicable on a scale of an hour or less. Plasma kinetic theory should be used on the smallest scale of seconds or less because the dimensions become comparable to the proton gyroradius. The concept of scale size is important and Burlaga and Ness (1967) have introduced the terms macroscale, mesoscale and microscale for scales on the order of 1 year ( $10^{4}$ hour), 4 days ( $10^{2}$ hour), and one hour, respectively. This definition is illustrated in Figure 5 where a fourth scale, the kinetic scale $\left(10^{-2}\right.$ hour), is introduced. Since the interplanetary plasma is convected at a speed of $\approx 400 \mathrm{~km} / \mathrm{sec}$. near the earth, it is possible to convert the time scale to a distance scale. Thus the macroscale, $10^{3}-10^{4}$ hour, corresponds to $10-10^{2} \mathrm{AU}$, the mesoscale corresponds to $1 \mathrm{AU}$, the microscale corresponds to $10^{-2} \mathrm{AU}$, and the kinetic scale corresponds to $10^{-4} \mathrm{AU}$ or $1.5 \times 10^{4} \mathrm{~km}$. Figure 5 also shows a characteristic frequency associated with each scale. This is simply the reciprocal of the characteristic time. The characteristic energy in Figure 5 is the energy of a proton whose gyroradius in a $5 y$ field is equal to the scale length. The sectors, spiral structure, and randomness in Figure 1 are macroscale phenomena. The structure behind interplanetary shock waves, which we 
shall not discuss, and the distribution of discontinuities between the earth and sun (previously referred to as the "filamentary" structure) are mesoscale phenomena. Tangential discontinuities, D-sheets, and waves can be seen on the microscale. Kinetic scale structures are such features as current sheets and hydromagnetic shocks.

\section{DISTRIBUTION OF DIRECTIONAL DISCONTINUITIES}

A. Definition of a Directional Discontinuity

Microscale discontinuities in the direction of the interplanetary magnetic field are shown in Figure 3. It is obvious that an analysis of the distribution of such discontinuities requires a clear definition of a discontinuity. Moreover, in order to scan the large amount of data which is available, this must be a quantitative definition which can be programmed for a computer. We shall define a directional discontinuity in terms of the $30 \mathrm{sec}$ averages of the Pioneer 6 measurements. The procedure is as follows:

1) A pair of successive 30 sec average data points $\left(\phi_{1}, \theta_{1}\right)$ and $\left(\phi_{2}, \theta_{2}\right)$ is selected, and the angle $\omega$ between the two corresponding magnetic field vectors is computed from the equation

$$
\cos \omega=\cos \theta_{1} \cos \theta_{2} \cos \left(\phi_{1}-\phi_{2}\right)+\sin \theta_{1} \sin \theta_{2}
$$

If $\omega \leq 30^{\circ}$, the process is repeated for the next pair of data points. This continues until a pair is found for which $\omega>30^{\circ}$. Such a pair is isolated for further analysis, and the process continues throughout a specified time interval until all such discontinuities have been isolated. 
2) Next, $\omega$ is recomputed by the following method. Consider a discontinuity between successive 30 sec averages $A$ and $B$. An average of $\phi$ and $\theta$ is computed for the two points preceding $A$ and for the two points following B, i.e.,

$$
\begin{array}{ll}
\bar{\phi}_{1}=\left(\phi_{\mathrm{A}-1}+\phi_{\mathrm{A}-2}\right) / 2, & \bar{\phi}_{2}=\left(\phi_{\mathrm{B}+1}+\phi_{\mathrm{B}+2}\right) / 2 \\
\bar{\theta}_{1}=\left(\theta_{\mathrm{A}-1}+\theta_{\mathrm{A}-2}\right) / 2, & \bar{\theta}_{2}=\left(\theta_{\mathrm{B}+1}+\theta_{\mathrm{B}+2}\right) / 2 ;
\end{array}
$$

then $\bar{\omega}$ is computed by substituting the se averages into Equation 1 . Discontinuities are rejected if $\bar{\omega}<30^{\circ}$. This procedure eliminates noise points and highly disturbed regions. Approximately $30 \%$ of the discontinuities from Step 1 are eliminated by this procedure.

3) The discontinuities obtained by Step 1 fall into several classes which are illustrated in Figure 6. The singles are most common and are most nearly consistent with the intuitive picture of a discontinuity. A single is simply a $30^{\circ}$ change in field direction which occurs in 30 seconds and which is not preceded or followed by another discontinuity within 2 minutes. Approximately $20 \%$ of the discontinuities obtained in Step 1 occur in pairs, i.e., two discontinuities occur separated by a 30 second interval. Such doubles, illustrated in Figure 6, can be considered as a single discontinuity. A double would be seen if a true discontinuity occurred during a 30 second average of the field quantities. Thus, we shall regard both singles and doubles as true discontinuities. Note that the 
method of computing $\bar{\omega}$ by using (1) and (2) gives two values of $\bar{\omega}$ for a double: one computed from the ovals in Figure 6 and one from the squares. These two values of $\bar{\omega}$ for a double are usually found to be equal within $15^{\circ}$; if they are not, the double is rejected. Triples (see Figure 6) and higher multiples are also observed, but they are rare; less than 5\% of the discontinuities obtained in Step 1 are multiples. A multiple could be the result of several closely spaced discontinuities or a large gradient. Multiples are eliminated from the set of discontinuities obtained in Step 1. We also disregard discontinuities which are separated by an interval of less than 2 minutes; this eliminates another $5 \%$ of the discontinuities from Step 1.

4) As a final check, these discontinuities are individually inspected in microscale plots, and ambiguous discontinuities are eliminated. This subjective procedure eliminates only $10 \%$ of the discontinuities, so it does not introduce an appreciable error.

The result of applying the procedure in the 4 above steps is a set of directional discontinuities. Figure 7 illustrates the variety of discontinuities obtained by this procedure. Time is given on the horizontal scale; each of the points in this Figure is a 30 second average. The case $\bar{\omega}=154^{\circ}$ shows a particularly clear discontinuity. The cases $\bar{\omega}=35^{\circ}, \bar{\omega}=43^{\circ}$ and $\bar{\omega}=47^{\circ}$ are more typical; there are variations in the field direction on either side of the discontinuity, but the discontinuity does stand out clearly in each case. The cases $\bar{\omega}=109^{\circ}$ and 
$\bar{\omega}=95^{\circ}$ each show a large discontinuity marked by a dashed line and a discontinuity with $\bar{\omega}<30^{\circ}$ which is not marked; discontinuities with $\bar{\omega}<30^{\circ}$ are not uncommon, but they are not considered here because it is frequently difficult to separate them from fluctuations and noise. The cases $\bar{\omega}=120^{\circ}$ and $\bar{\omega}=95^{\circ}$ illustrate accepted discontinuities which are marginal in terms of the definition which was given above.

\section{B. Time Distribution of Directional Discontinuities}

The procedure described above has been applied to identify directional discontinuities which passed the Pioneer 6 spacecraft during the interval December 16, 1965-January 4, 1966. The results are shown in Figure 8 where each discontinuity is indicated by a line whose height is equal to $\bar{\omega}$ and whose position on the abscissa gives the time at which the discontinuity was observed. Five graphs are shown in Figure 8, each having a time scale of four days. Thus, assuming a $400 \mathrm{~km} / \mathrm{sec}$ solar wind, the length of the abscissa in each graph corresponds to a characteristic length of $1 \mathrm{AU}$. The shaded area in these graphs represent intervals for which high time resolution data are not available. A number of interesting qualitative results can be seen immediately from Figure 8 .

1) Discontinuities are present throughout the 20-day period.

2) There are "quiet" days with $\approx 10$ discontinuities per day (e.g., day 351) and there are "disturbed" days with $\approx 50$ discontinuities per day (e.g., day 363$)$.

3) Most discontinuities have $\bar{\omega}<90^{\circ}$, but there are several discontinuities with $\bar{\omega}>135^{\circ}$. 
4) There is a tendency for discontinuities to occur in clusters consisting of several discontinuities per hour, and there are intervals up to several hours which contain no directional discontinuities.

We shall now examine points $1-4$ in detail.

Consider the distribution of the discontinuities in Figure 8 as a function of $\bar{\omega}$. This distribution was obtained by simply counting the number of discontinuities which fell in the intervals $30^{\circ}<\bar{\omega} \leq 60^{\circ}, 60^{\circ}<\bar{\omega} \leq 90^{\circ}$, etc. The result is shown in Figure 9. It is evident that the number of discontinuities falls off rapidly as a function of $\bar{\omega}$. In fact, the distribution can be fitted by the function $\mathrm{e}^{-\left(\omega / 75^{\circ}\right)^{2}}$. This function is empirical and somewhat arbitrary, but it is interesting that this is the same function which Burlaga and Ness (1968) used to fit the corresponding distribution for a different set of directional discontinuities. This set consists of 76 clear discontinuities which were selected from $\approx 2500$ hours of data for the period December 16, 1965 to June 1966; the definition of these discontinuities was somewhat different than that used in this paper. The fact that the same function fits $\mathrm{dN} / \mathrm{d} \omega$ for these discontinuities as well as the discontinuities in Figure 8 suggests that the distribution is not sensitive to details of the definition of a directional discontinuity and that the distribution is representative of average conditions during the first half of 1966. An extrapolation of the distribution in Figure 9 suggests that there are numerous discontinuities with $\bar{\omega} \leq 30^{\circ}$, so the density of discontinuities may be much higher than appears in Figure 8 which shows only discontinuities with $\bar{\omega}>30^{\circ}$. 
Let us return to the time distribution of the discontinuities in Figure 8. Since discontinuities with $\bar{\omega} \leq 30^{\circ}$ cannot always be identified, the time distribution of all discontinuities cannot be computed. Therefore, the discontinuities were divided into four sets $-30^{\circ}<\bar{\omega} \leq 60^{\circ}, 60^{\circ}<\bar{\omega} \leq 90^{\circ}, 90^{\circ}<\bar{\omega} \leq 120^{\circ}, 120^{\circ}<\bar{\omega}$ $\leq 150^{\circ}$-and the distribution was computed for each set. For example, consider the set $30^{\circ}<\bar{\omega} \leq 60^{\circ}$. Discontinuities in this set were isolated, and the time intervals $\Delta T_{i}$ between successive discontinuities were computed. The distribution of these intervals was then determined by simply counting the number of intervals with $0<\Delta \mathrm{T}_{\mathrm{i}} \leq .04$ days, $.04<\Delta \mathrm{T}_{\mathrm{i}} \leq .08$, etc. The same procedure was repeated for the other sets of discontinuities using different intervals for the $\Delta \mathrm{T}$ scale. The resulting distributions are shown in Figure 10. Most notable is the approximately exponential dependence of the number of intervals as a function of $\Delta \mathrm{T}$ with most discontinuities tending to be separated by a relatively small interval. The clustering in Figure 8, which was noted earlier, is simply a manifestation of this exponential dependence. The form of the distributions is what would be expected if discontinuities occur with a Poisson distribution. The decreasing amplitudes and the smaller slopes of the curves for larger $\bar{\omega}$ in Figure 10 are a consequence of the distribution shown in Figure 9. From the curves in Figure 10, it is possible to compute the mean time separation of the discontinuities in each set. Assuming that the discontinuities are convected past the spacecraft at $\approx 400 \mathrm{~km} / \mathrm{sec}$, a mean spatial separation can be computed for the discontinuities in each set. These separations are shown in 
Figure 11. The most abundant discontinuities $\left(30^{\circ}<\bar{\omega} \leq 60^{\circ}\right)$ have a mean separation $\approx .02 \mathrm{AU}$. Larger discontinuities $\left(120^{\circ}<\bar{\omega} \leq 150^{\circ}\right)$ are separated by $\approx .1 \mathrm{AU}$. These numbers are means for the 20 day interval shown in Figure 8.

\section{Other Characteristics of Directional Discontinuities}

Let $B_{1}$ be the magnetic field intensity just before a directional discontinuity arrives at the spacecraft, and let $B_{2}$ be the magnetic field intensity just after the discontinuity passes the spacecraft. It is of interest to know how $B_{1}$ and $B_{2}$ are related. The quantity $\left(\mathrm{B}_{1}-\mathrm{B}_{2}\right) / \max \left(\mathrm{B}_{1}, \mathrm{~B}_{2}\right)$ was computed for each of the discontinuities in Figure 8 and the distribution of these quantities was determined. The result is shown in Figure 12. It is evident that for most discontinuities $B_{1} \approx B_{2}$, i.e., the magnetic field intensity usually does not change across a directional discontinuity. Only for $\approx 25 \%$ of the discontinuities does the magnetic field intensity change by more than $20 \%$.

For each directional discontinuity, it is possible to define a direction ${\underset{\sim}{B}}_{1} \times \underset{\sim}{\mathrm{B}_{2}} /\left(\mathrm{B}_{1} \times \mathrm{B}_{2}\right)$, where ${\underset{\sim}{\mathrm{Z}_{1}}}_{1}$ and ${\underset{\sim}{\mathrm{B}}}_{2}$ are the magnetic field vectors before and after a discontinuity. If a directional discontinuity is a tangential discontinuity, this direction is normal to the plane of the discontinuity. The distribution of such vectors which was computed for the discontinuities in Figure 8 is shown in Figure 13. The distributions in this figure were computed by putting all of the vectors in their proper orientations at the origin of a unit sphere and computing the density of the points which were determined by the intersections of these vectors with the surface of the sphere. The left side of Figure 13 shows the 
density, integrated over $\phi$, as a function of $\theta$. The right side shows the density, integrated over $\theta$, as a function of $\phi$. The radius of each sector in Figure 13 is proportional to the density for that interval. Note that the ecliptic plane components of $\underset{\sim}{B_{1}} \times \underset{\sim}{B_{2}}$ tend to be perpendicular to the macroscale spiral direction. Also, note the strong tendency to be directed away from the ecliptic plane. The bilateral symmetry of the distributions in Figure 13 and in Figure 12 expresses a statistical equivalence between $\underset{\sim}{\mathrm{B}}$ and $\underset{\sim}{\mathrm{B}}$.

\section{Relation Between Discontinuities and $\Sigma \mathrm{Kp}$}

It was noted that Figure 8 shows "quiet" periods with few discontinuities, and "disturbed" periods with a high density of discontinuities. It is natural to ask whether such periods are related to quiet and disturbed geomagnetic conditions as indicated by $2 \mathrm{Kp}$. This is answered in Figure 14 where the number of discontinuities for each of the days in Figure 8 is plotted versus $\Sigma \mathrm{Kp}$ for the corresponding days. The dots indicate that the data for that day were complete. Crosses indicate days with data gaps. For a day with incomplete data a density of discontinuities (number per hour) was computed, and the number of discontinuities for that day was taken to be this density times 24 hours. No attempt was made to correct for the separation between the earth and the spacecraft, which increased from essentially zero to $3 \times 10^{6} \mathrm{~km}$ during the period in question and may imply a time lag up to $\approx 2 \mathrm{hr}$ (Ness, 1966). Figure 8 shows that there tends to be more discontinuities during geomagnetic disturbed times. Of course, this does not imply a causal relation. 


\section{FILAMENTS AND FLUX TUBES}

It has already been noted, in connection with Figures 2 and 3 , that the two outstanding characteristics of the microscale interplanetary magnetic field are the existence of numerous discontinuities with a mean separation on the order of an hour and the common occurrence of intervals up to an hour or more during which the field direction is nearly constant. These characteristics can also be seen in Figure 15 and 16, which show a quiet and disturbed period, respectively. During the quiet interval, there are few directional discontinuities and the field direction generally changes relatively slowly on a scale of one hour. During the disturbed interval (Figure 16), there are numerous discontinuities (24/(20 hour)) and the field direction often changes by more than $60^{\circ}$ in an hour; but even here there are intervals during which the field direction remains relatively constant. The discontinuity at $0610 \mathrm{UT}$ is identified as a shock, since it was associated with a sudden-commencement at the earth. These two antithetical microscale components - the directional discontinuities, which were discussed in detail above, and intervals of rather constant field direction, which will be quantitatively discussed in a subsequent paper-combine to present a picture of the interplanetary medium which is markedly different from the "turbulent" macroscopic picture. The precise manner in which these two elements combine to form the mesostructure is a subject of considerable interest, which we shall discuss briefly . 
The earliest suggestion was that one could think of pairs of directional discontinuities between which the field was uniform and slowly varying (see the Introduction). One such aggregate was called a filament, and the mesoscale interplanetary magnetic field was considered to consist of a collection of intertwined filaments. The filamentary concept correctly emphasizes the two basic components of the field, but the general picture that it suggests can be very misleading. For example, where are the filaments in Figure 15? The question cannot be answered precisely for two reasons: 1) not all discontinuities can be identified because fluctuations are present, which can make it difficult to unambiguously pick out discontinuities; 2) the field direction is not always nearly constant between discontinuities. To put it another way, an assembly of filaments would imply that the field directions in Figures 15 and 16 could be represented by a series of step functions, but such a fit is not a good approximation, and a unique fit of this kind is not possible. Thus the concept of an aggregate of filaments should be used with caution. Rather than think in terms of filamentary tubes, implying an ensemble of pairs of discontinuities, it would be better to think of an ensemble of discontinuities themselves. Thus, it would be more appropriate to think of the interplanetary medium as discontinuous rather than filamentary. The discontinuities are embedded in a varietal medium in which the magnetic field direction usually changes slowly on a scale of one hour, but large gradients and fluctuations may also be seen, especially during disturbed times. 
In the qualitative model which was just proposed, filamentary tubes are not considered to be fundamental to the overall structure of the interplanetary medium. However, it is sometimes convenient to think of flux tubes, which are purely imaginary and have arbitrary boundaries. The concept of a flux-tube is based on that of a line of magnetic force. The boundary of a flux-tube is everywhere tangent to lines of force. The cross-sectional area of a flux-tube is arbitrary, and may range from the area enclosed by a low energy gyrating electron to that enclosed by a sector (Wilcox and Ness, 1966); it is most convenient to choose an area over which the field direction is essentially constant.

It is important to distinguish between flux-tubes and filaments. When a directional discontinuity passes the spacecraft, it is correct to say that the spacecraft sees one flux-tube and the other with a different orientation, but it is not always true that the spacecraft passes from one filament to another, for discontinuities can exist where filaments cannot be seen. Of course, two-flux tubes need not be separated by a discontinuity in $\underset{\sim}{\mathrm{B}}$.

\section{SUMMARY}

Discontinuities in the direction of the interplanetary magnetic field were observed by the deep-space probe, Pioneer 6 during the period December 16 , 1965-January 4, 1966. Individual discontinuities can be seen when viewed on the microscale $(\approx 1$ hour $\approx .01 \mathrm{AU})$ and numerous discontinuities can be seen on the mesoscale $\approx 4$ days $\approx 1 \mathrm{AU})$. They are not observed on the macroscale $\approx 1$ year) and sometimes appear to be broad on the kinetic scale $\approx .5 \mathrm{~min}$ ). The 
directional discontinuities have a mean separation of approximately one hour, but there are days which the nụmber of discontinuities is a factor of two greater or smaller. The number of discontinuities per day tends to increase with $\Sigma \mathrm{Kp}$. The distribution of time intervals between directional discontinuities is approximately exponential, the small intervals being most probable.

Let ${\underset{\sim}{B}}_{1}$ and ${\underset{\sim}{\mathrm{B}}}_{2}$ be the magnetic field vectors just before and after a discontinuity. The distribution of discontinuities as a function of $\omega$, where $\omega$ is the angle between ${\underset{\sim}{\mathrm{B}}}_{1}$ and $\underset{\sim 2}{\mathrm{~B}}$, is proportional to exp $\left[-\left(\omega / 75^{\circ}\right)^{2}\right]$. The magnitude of the field usually does not change appreciably across a directional discontinuity; the change in $|\underset{\sim}{\mathrm{B}}|$ is less than $20 \%$ for $75 \%$ of the discontinuities. The vectors $\vec{B}_{1} \times \vec{B}_{2}$ tend to be directed out of the ecliptic plane and normal to the spiral direction. These distributions for the period December 16, 1965-January 4, 1966 are very similar to those which were found for a smaller set of discontinuities which were selected from the interval December 16, 1965-June 1966.

Directional discontinuities do not always form boundaries of filaments. In fact, it is shown that filaments are often difficult to identify and that the picture of an assembly of adjacent filaments does not adequately describe the interplanetary magnetic field. The interplanetary medium should be regarded as discontinuous rather than filamentary.

The origin of directional discontinuities is not yet known. The model of twisted filaments implies that the discontinuities originate at the sun. The model of a discontinuous medium at $\approx 1$ AU allows the possibility that discontinuities develop in the interplanetary medium. 


\section{ACKNOWLEDGMENTS}

The magnetic field data for this study were provided by Dr. Ness. I wish to acknowledge many fruitful discussions with Dr. Ness, and I thank Drs. D. H. Fairfield and K. W. Ogilvie for many helpful comments concerning the manuscript. 


\section{REFERENCES}

Bartley, W. C., Bukata, R. P., McCracken, K. G. and Rao, J. R.: 1966, Anisotropic Cosmic Radiation Fluxes of Solar Origin, J. Geophys. Res., $\underline{71}, 3297$. Burlaga, L. F.: 1968, Microscale Structures in the Interplanetary Medium, Solar Physics (in press).

Burlaga, L. F. and Ness, N. F.: 1968, Macro and Micro Structure of the Interplanetary Magnetic Field, Can. J. Phys., (in press).

Colburn, D. S. and Sonett, C. P.: 1966, Discontinuities in the Solar Wind, Space Sci. Rev. $\underline{5}, 439-506$.

McCracken, K. G. and Ness, N. F.: 1966, The Collimation of Cosmic Rays by the Interplanetary Magnetic Field, J. Geophys. Res., 71, 3315.

Ness, N. F.: 1966, Simultaneous Measurements of the Interplanetary Magnetic Field, J. Geophys. Res., $\underline{71,} 3319$.

Ness, N. F., Scearce, C. S., and Cantarano, S.: 1966, Preliminary Results from the Pioneer 6 Magnetic Field Experiment, J. Geophys. Res., 71, 3305.

Parker, E. N.: 1963, Interplanetary Dynamical Processes, Interscience Publishers, New York.

Siscoe, G. L., Davis, Jr., L., Coleman, Jr., P. J., Smith, E. J. and Jones, D. E.: 1968, Power Spectra and Discontinuities of the Interplanetary Magnetic Field: Mariner 4, J. Geophys. Res., $\underline{73}, 61$.

Wilcox, J. M. and Ness, N. F.: 1966, Quasi-Stationary Corotating Structure in the Interplanetary Medium, J. Geophys. Res., 70, 5793. 


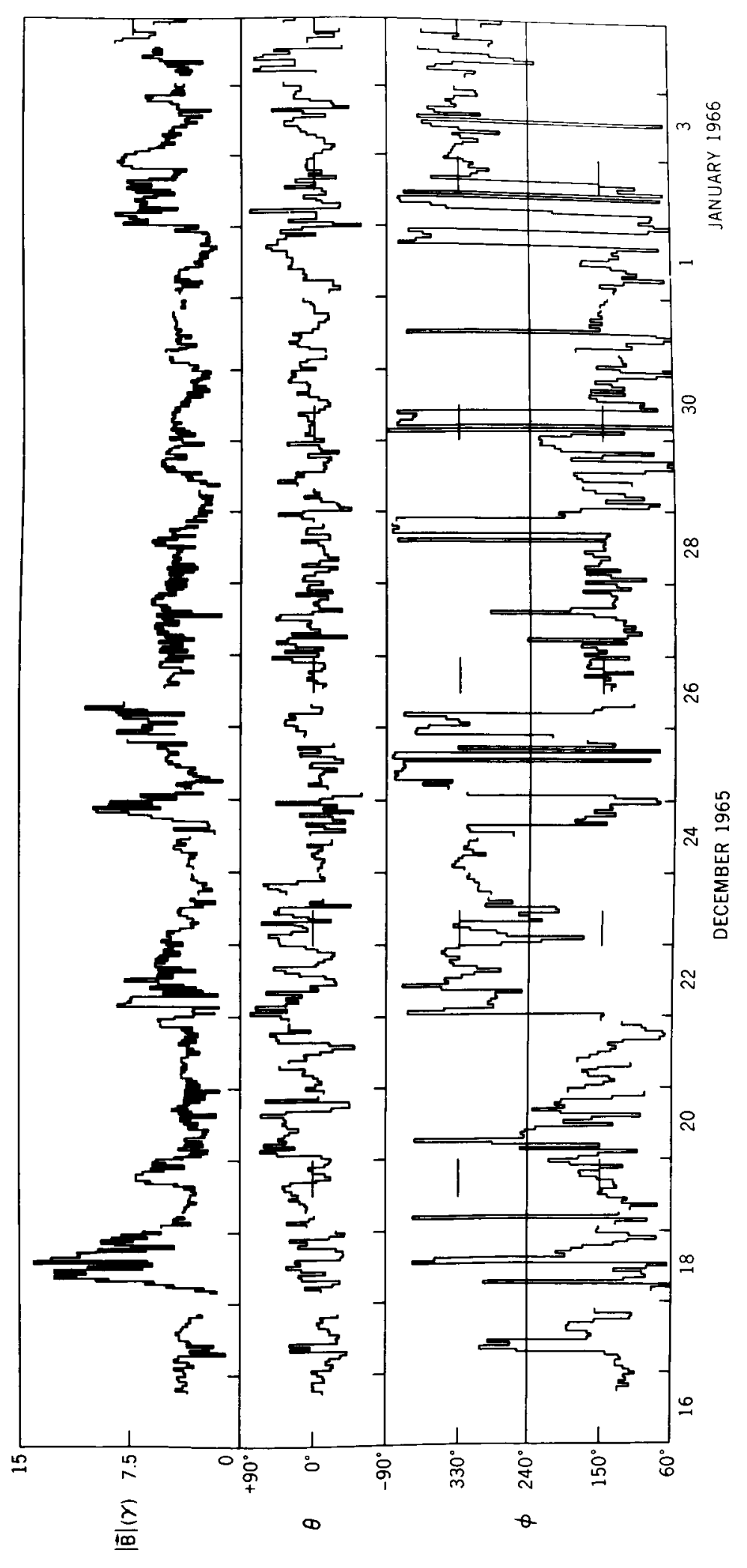

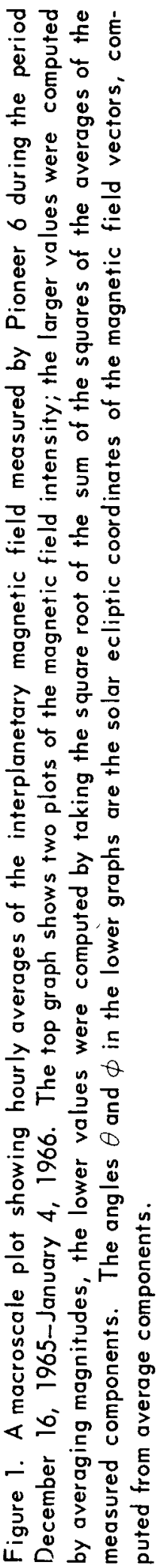




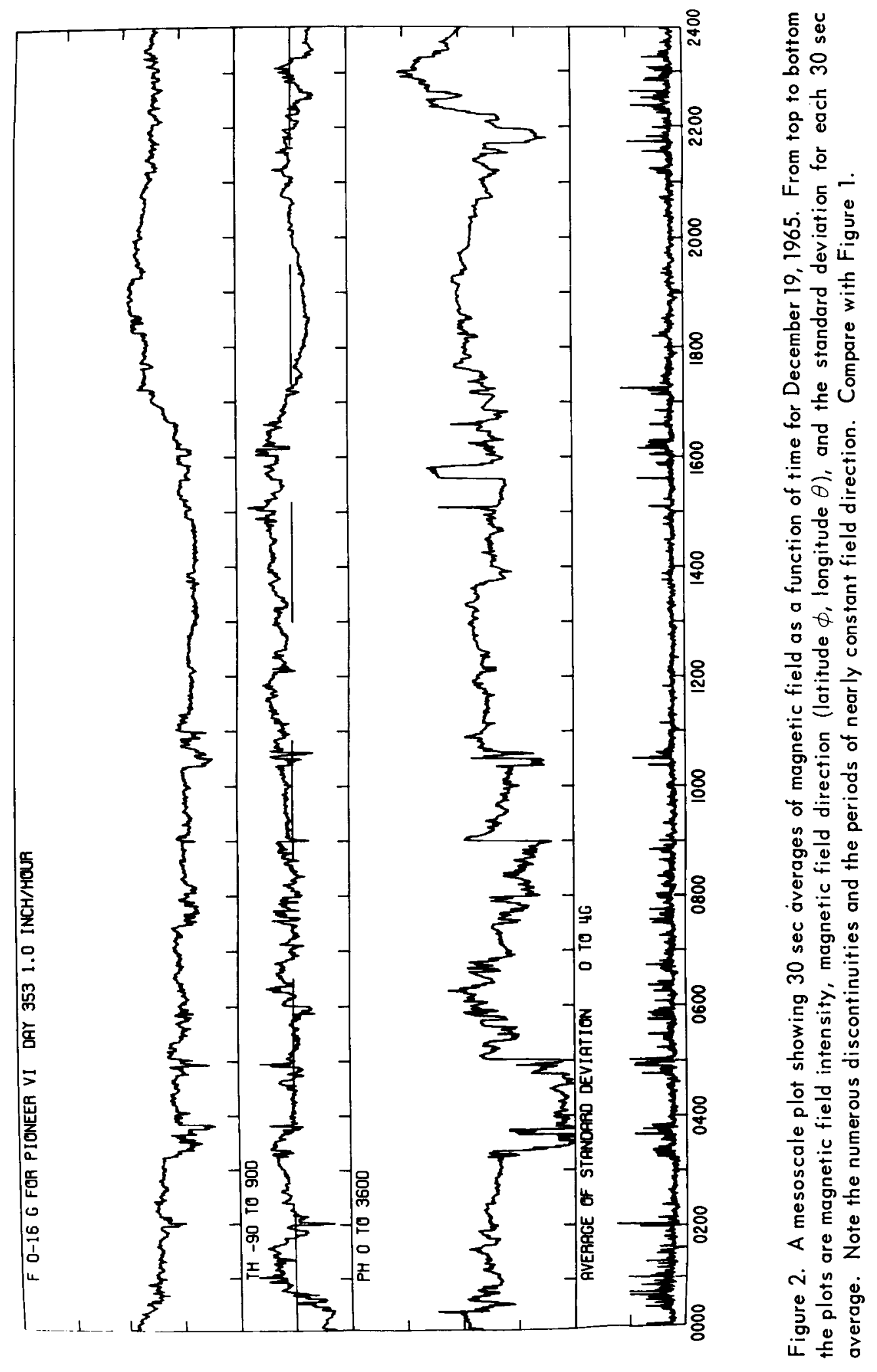




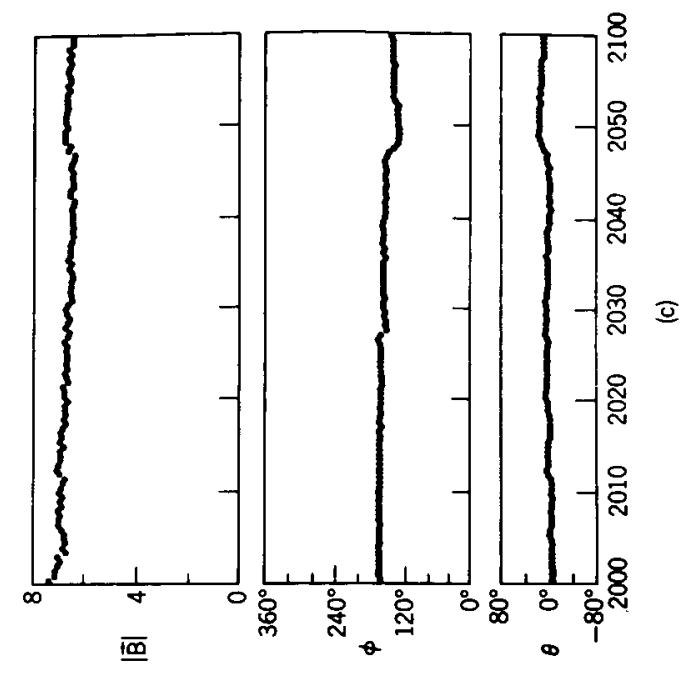

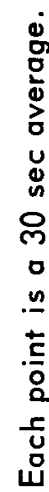
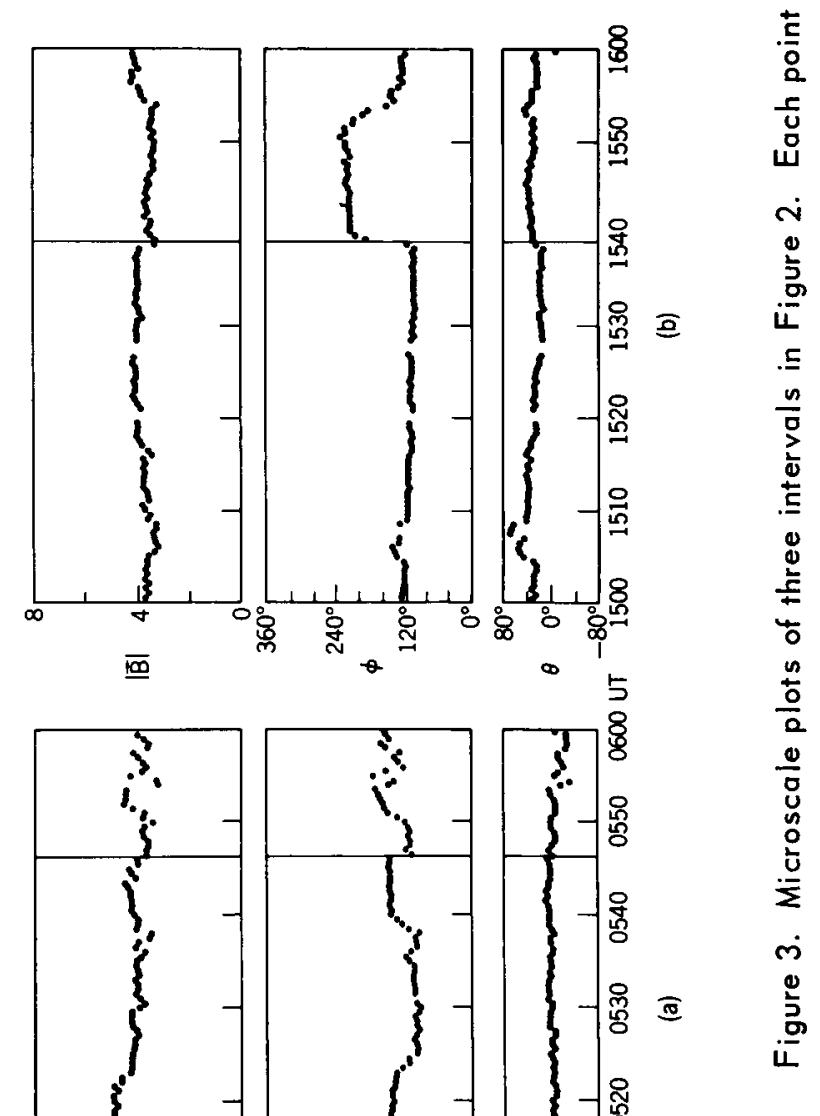


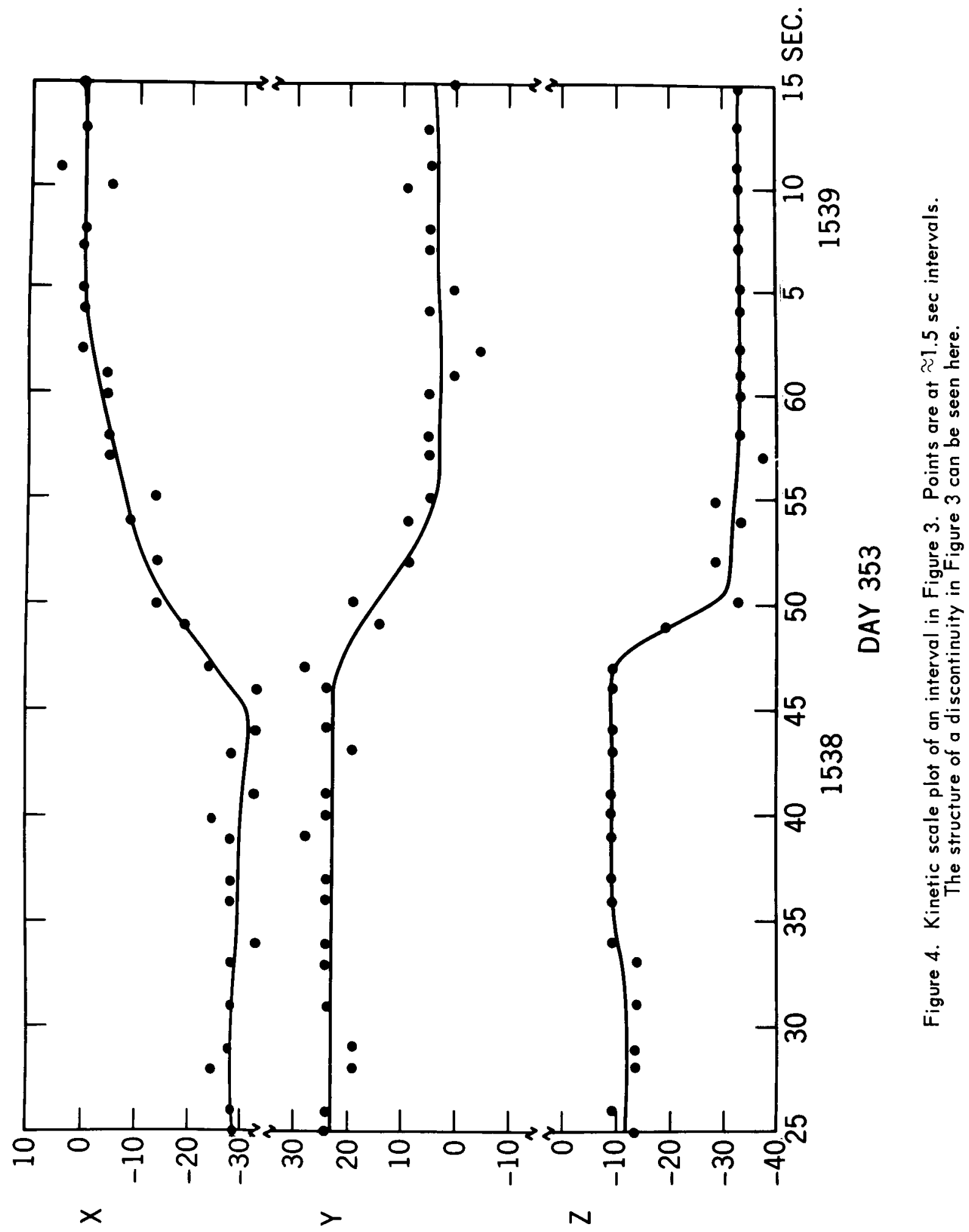




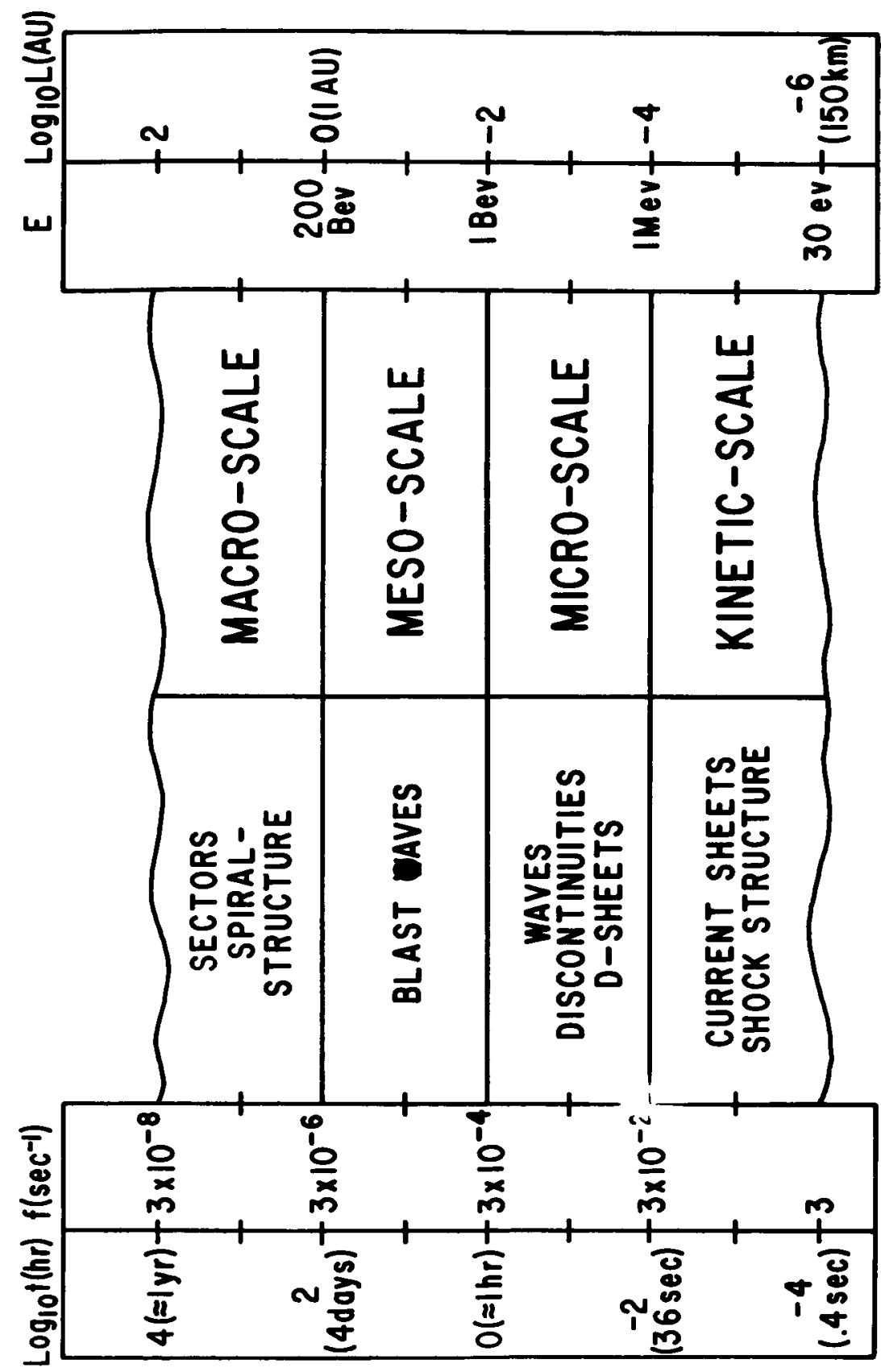

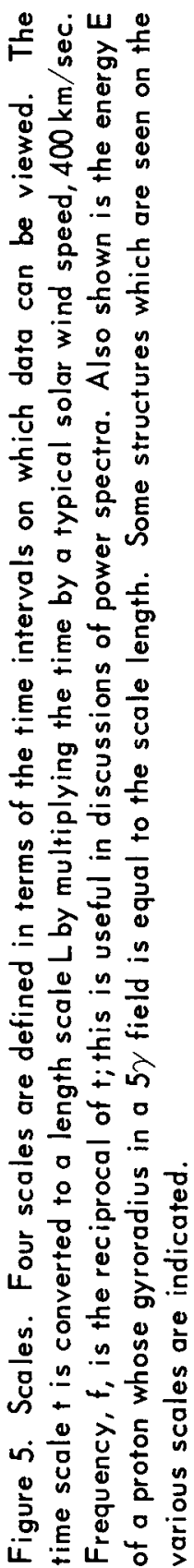




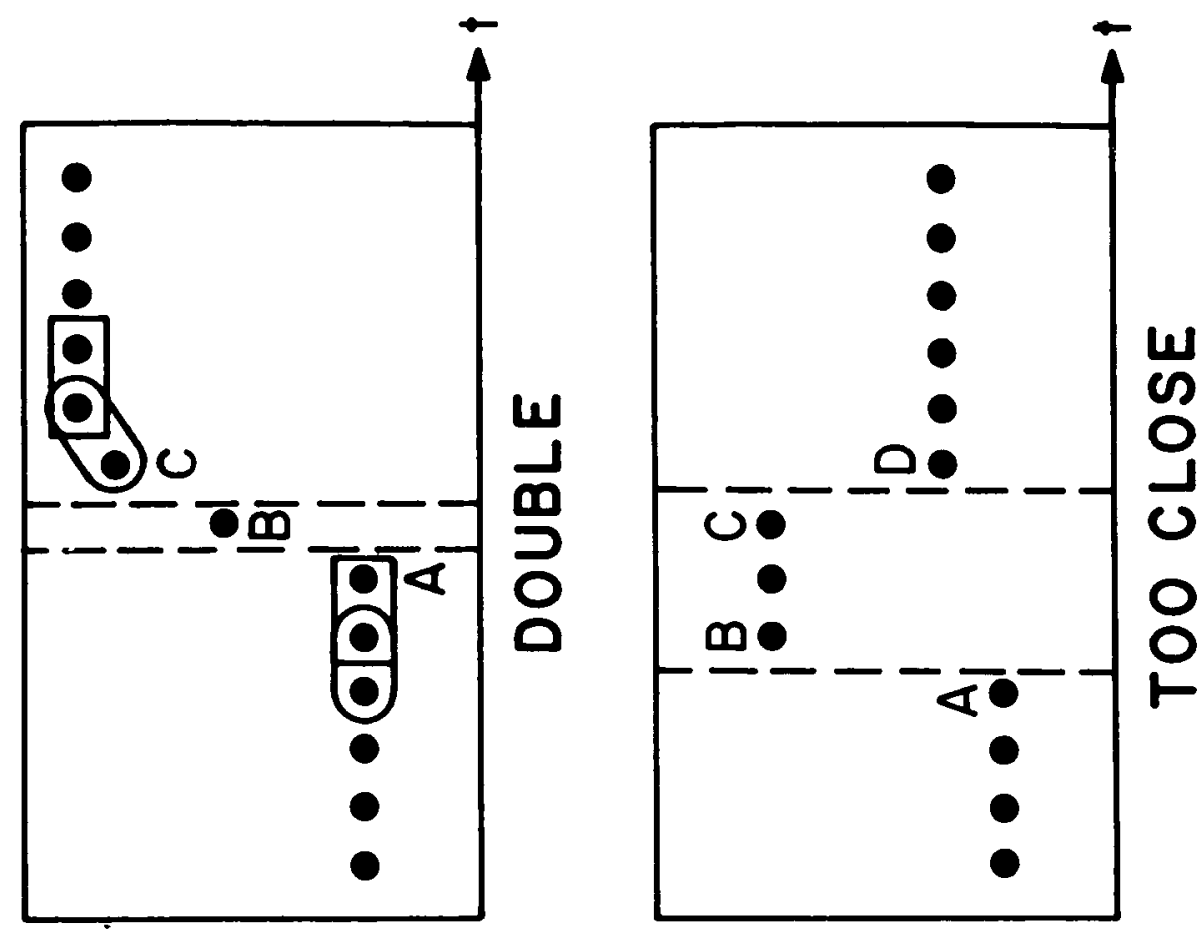

웅

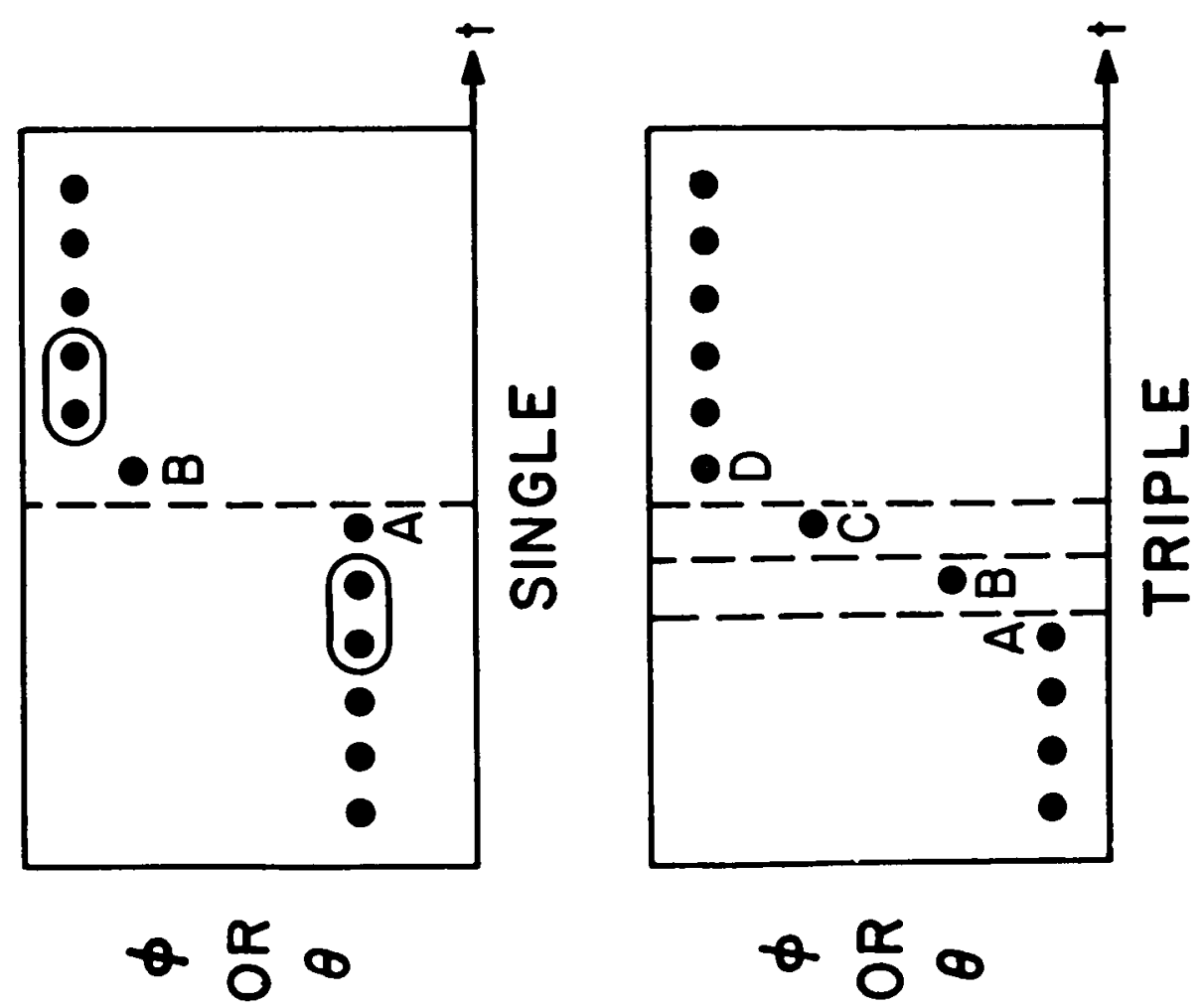

$\theta \div \frac{5}{ㅇ ㅡ ㅇ ~}$

$\div \frac{5}{3}$

$\frac{5}{n} \mathbb{E}$

。客U

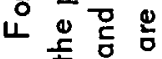

$\dot{\theta} \div$

o

D我这

० ว

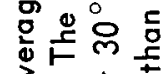

वे

प⿺辶⿻二口犬

ติ

유요.르

도 융

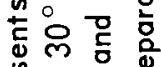

ڤ $\wedge$

훙현

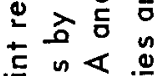

옹

돈

山ั

. 음

준웅

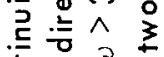

웅

远.

능

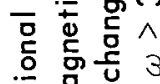

¿

일

le) 0 范

뭉ㅎㅀ

돈

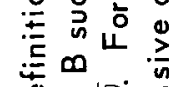

8100

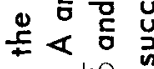

다의

喜迹吉分

站

至。

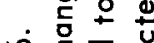

넌웡 =

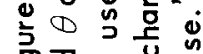

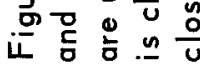



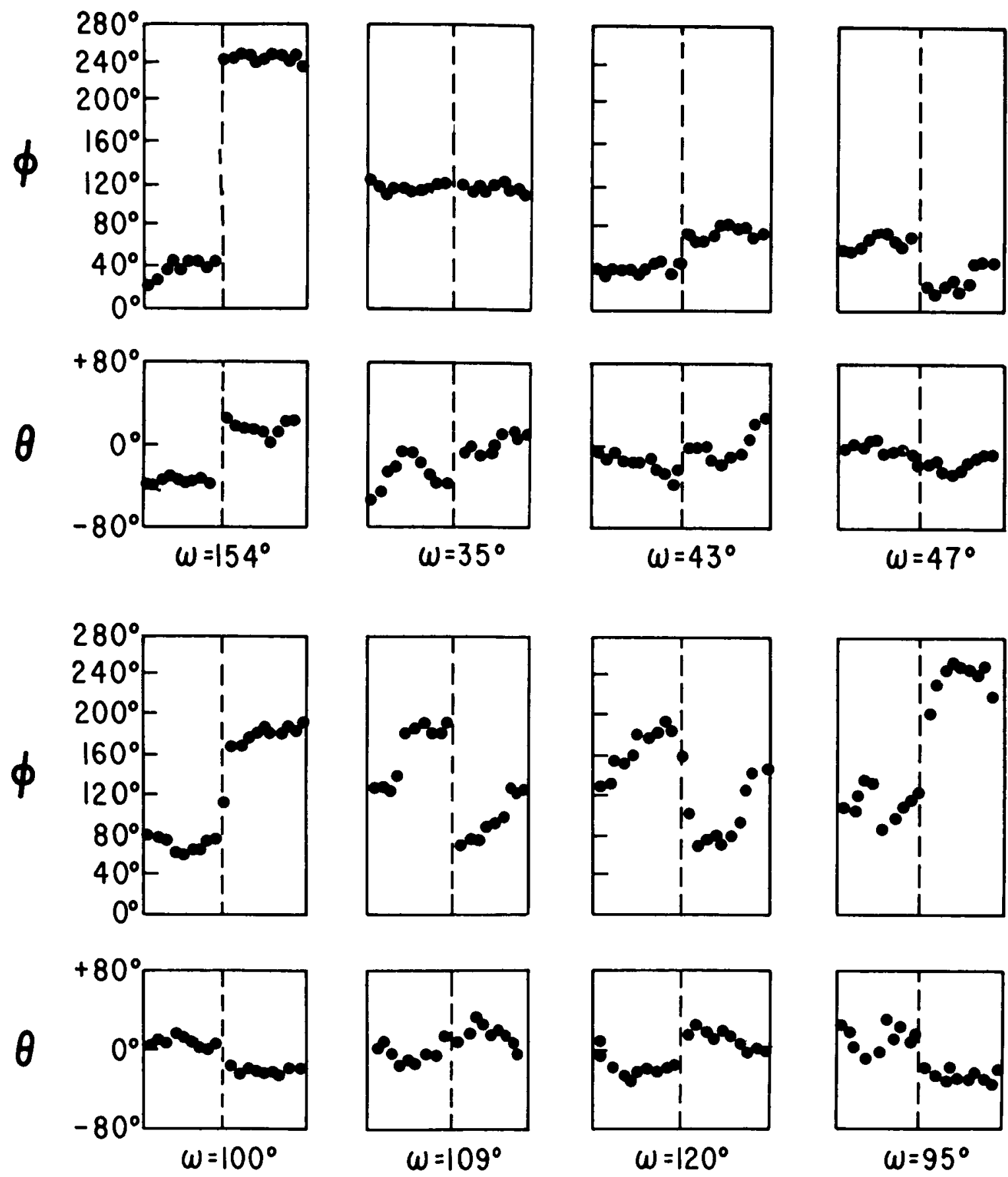

Figure 7. Examples of directional discontinuities. 


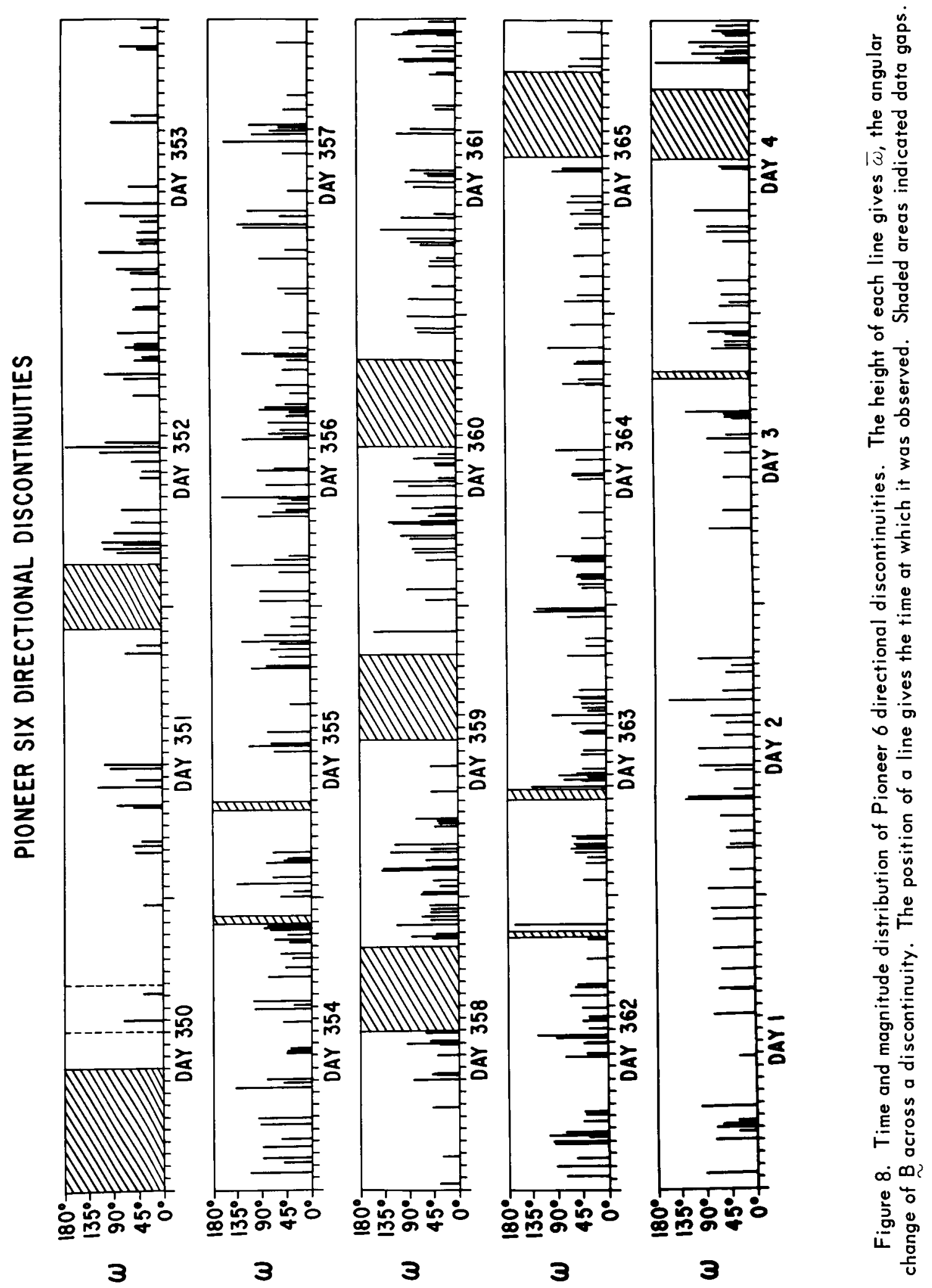




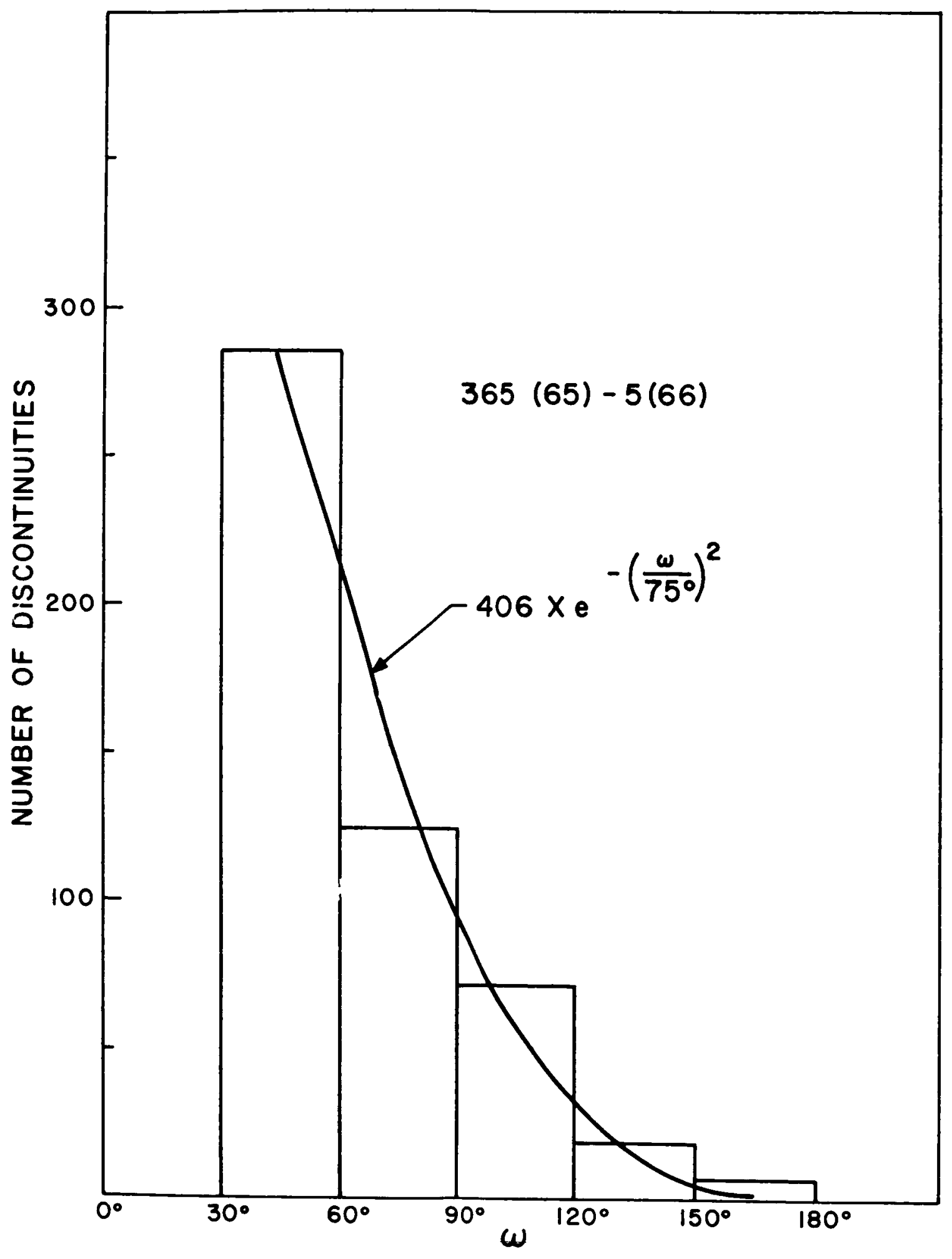

Figure 9. Distribution of the directional discontinuities in Figure 8 as a function of $\bar{\omega}$. 

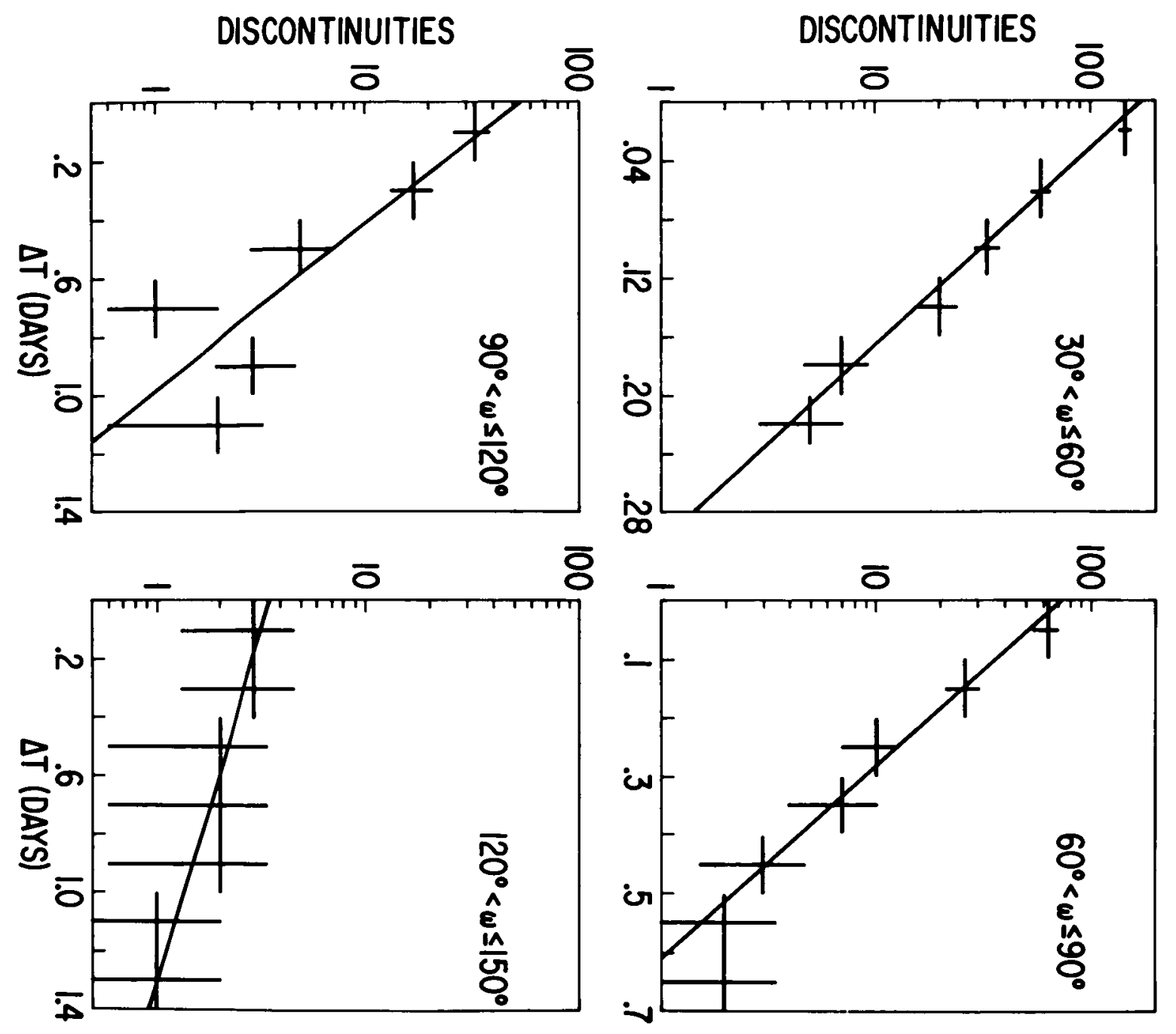

Figure 10. Distribution of time intervals between successive directional discontinuities, (see text). 


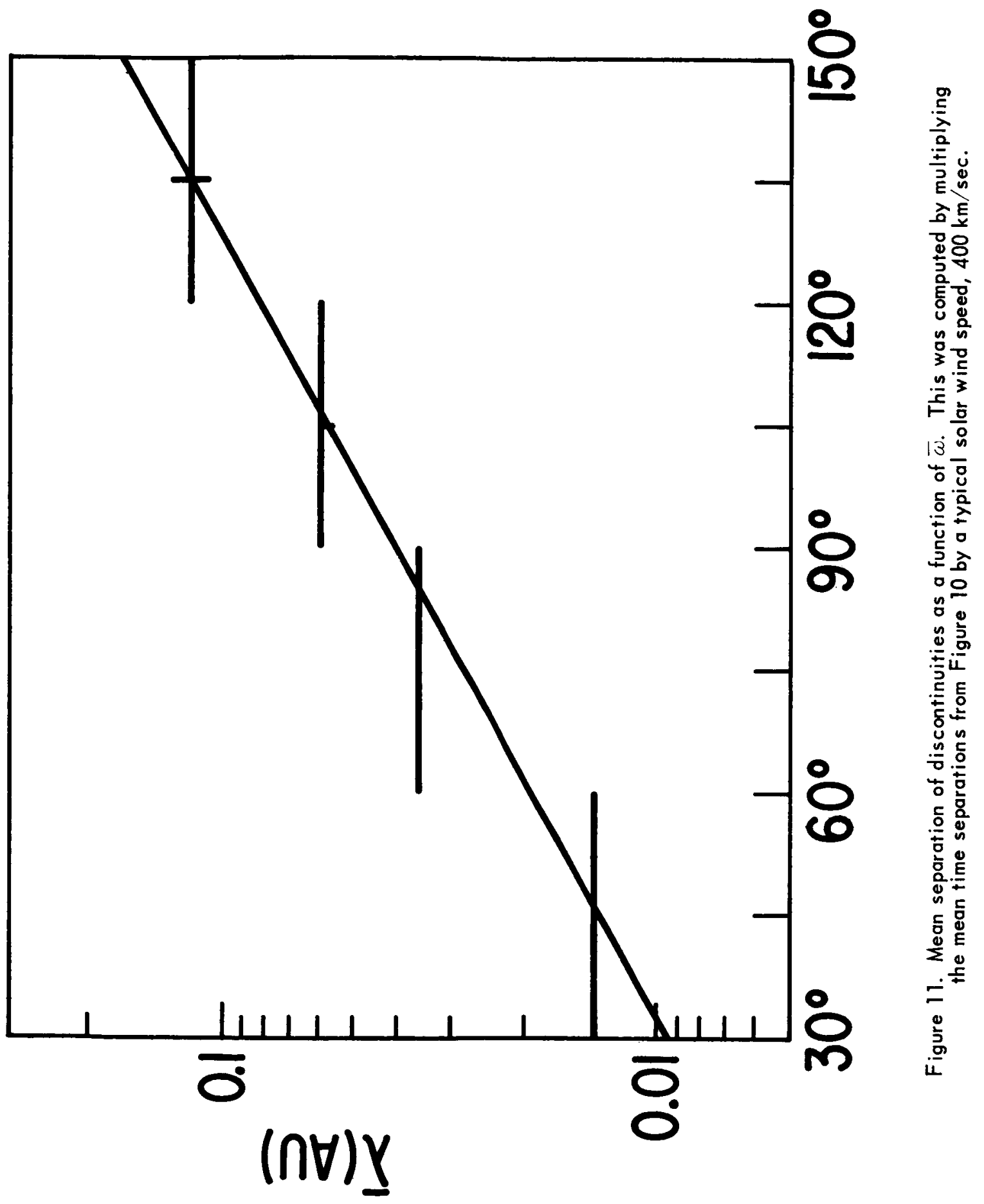




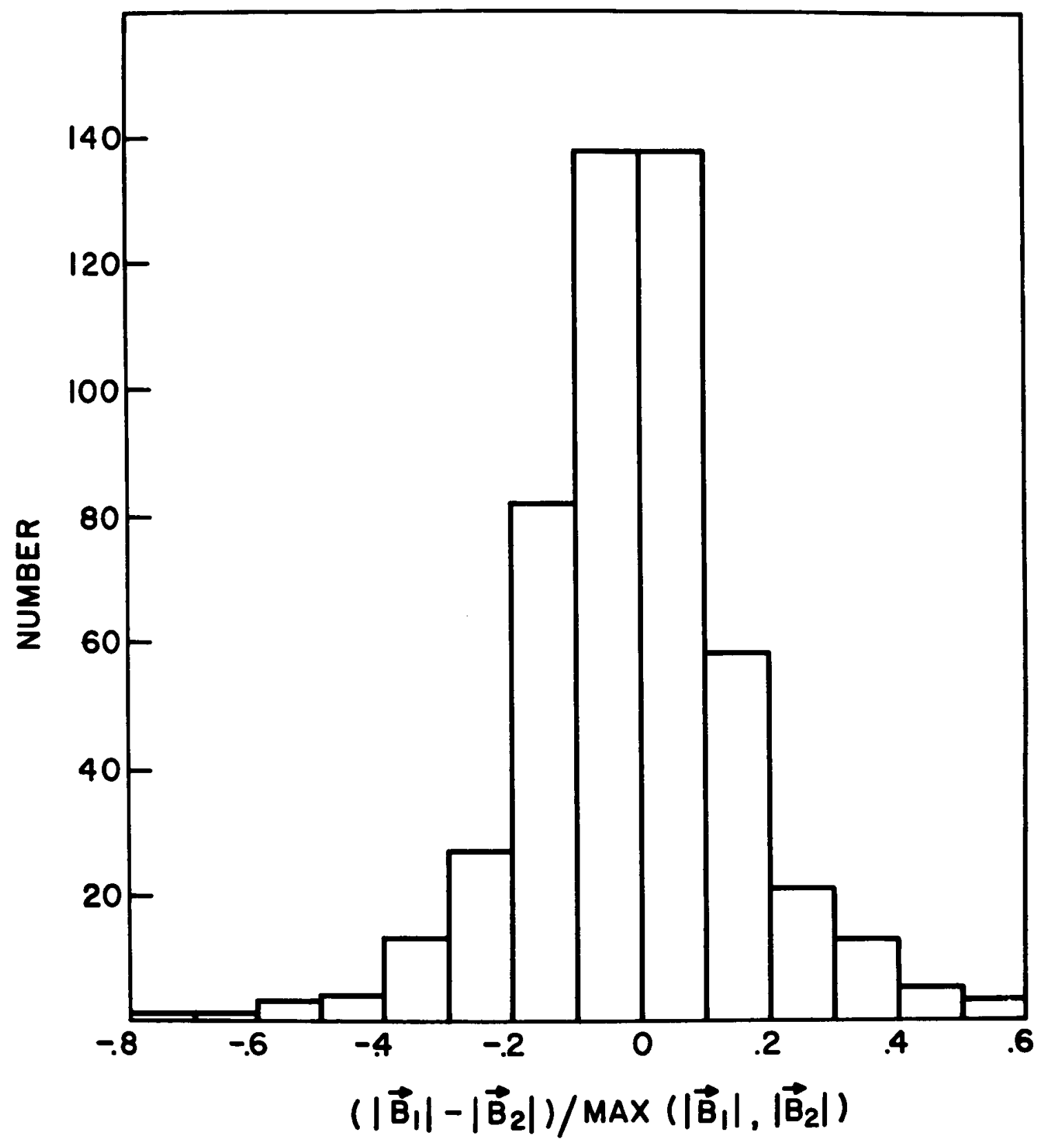

Figure 12. Distribution of changes in $|\underset{\text { B }}{\mathbf{B}}|$ ocross the directional discontinuities in Figure 8. 


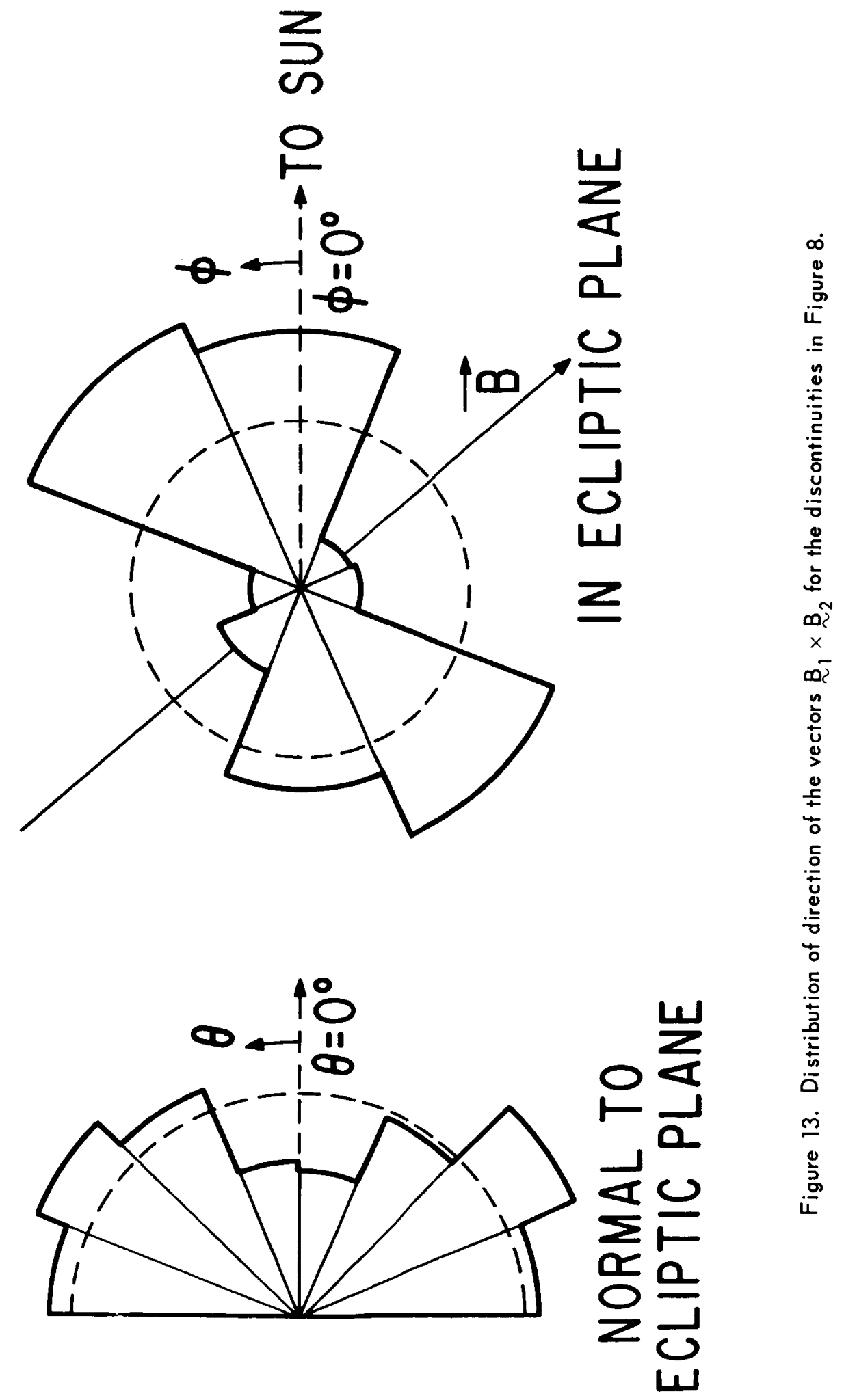




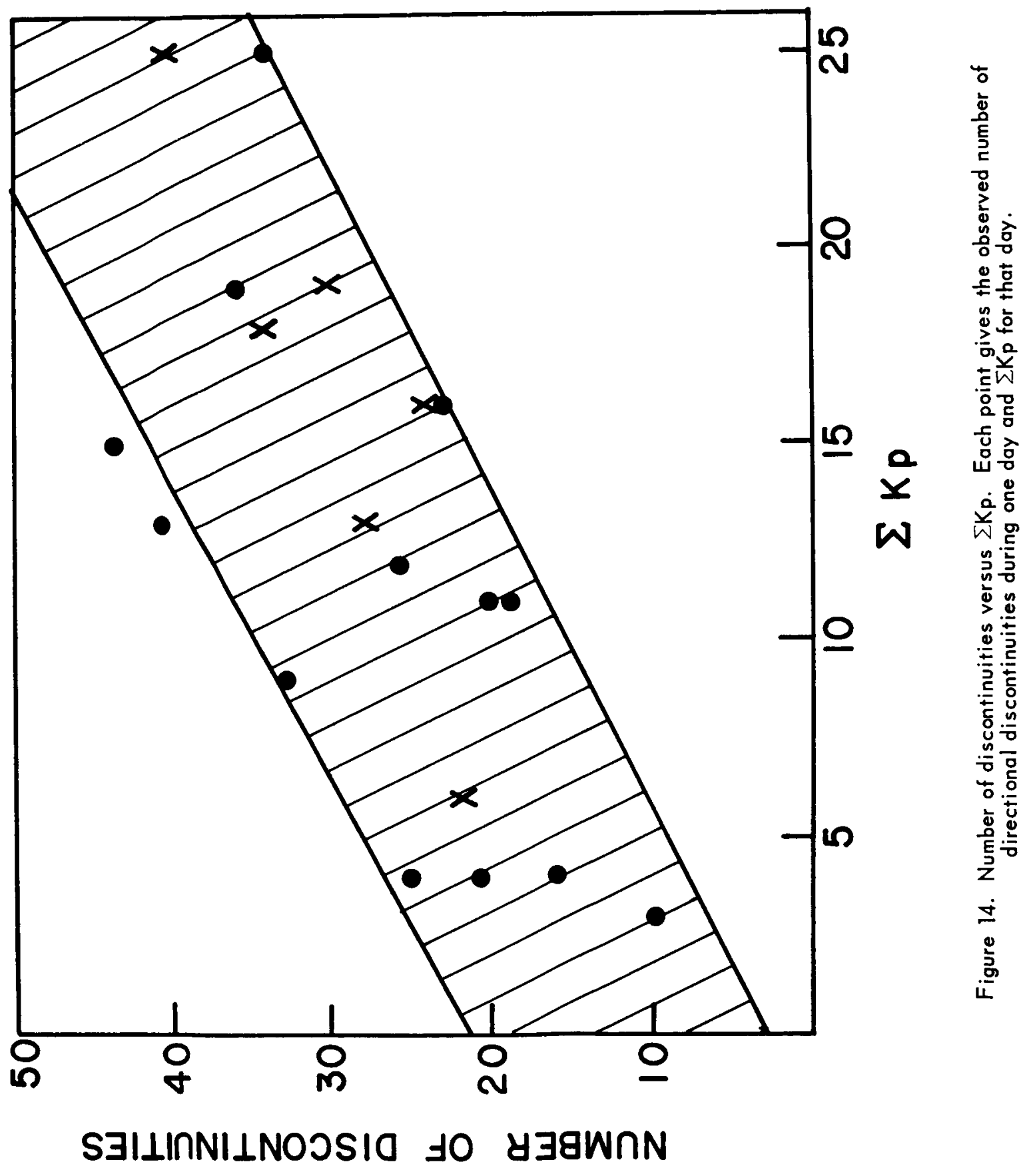




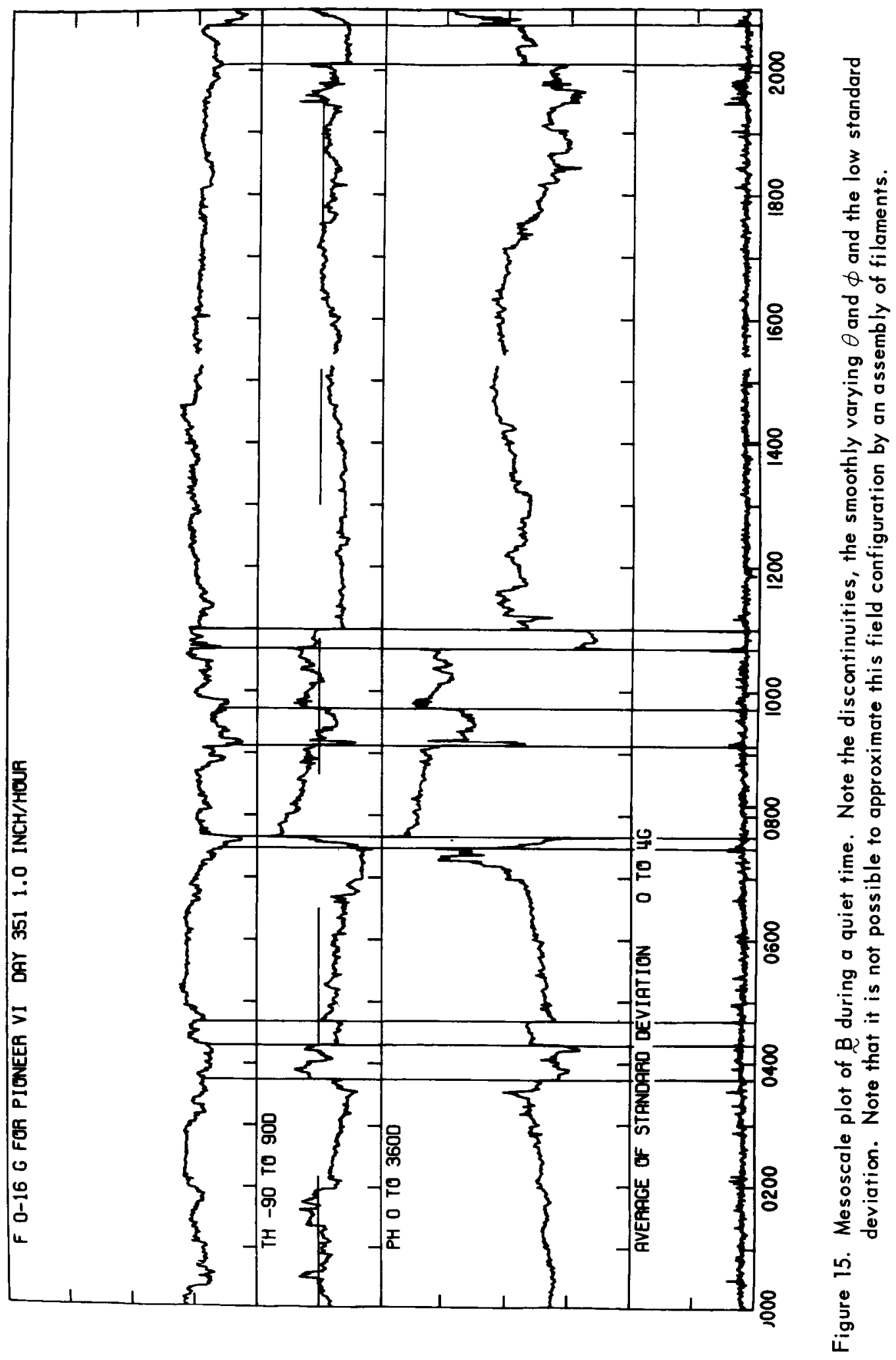




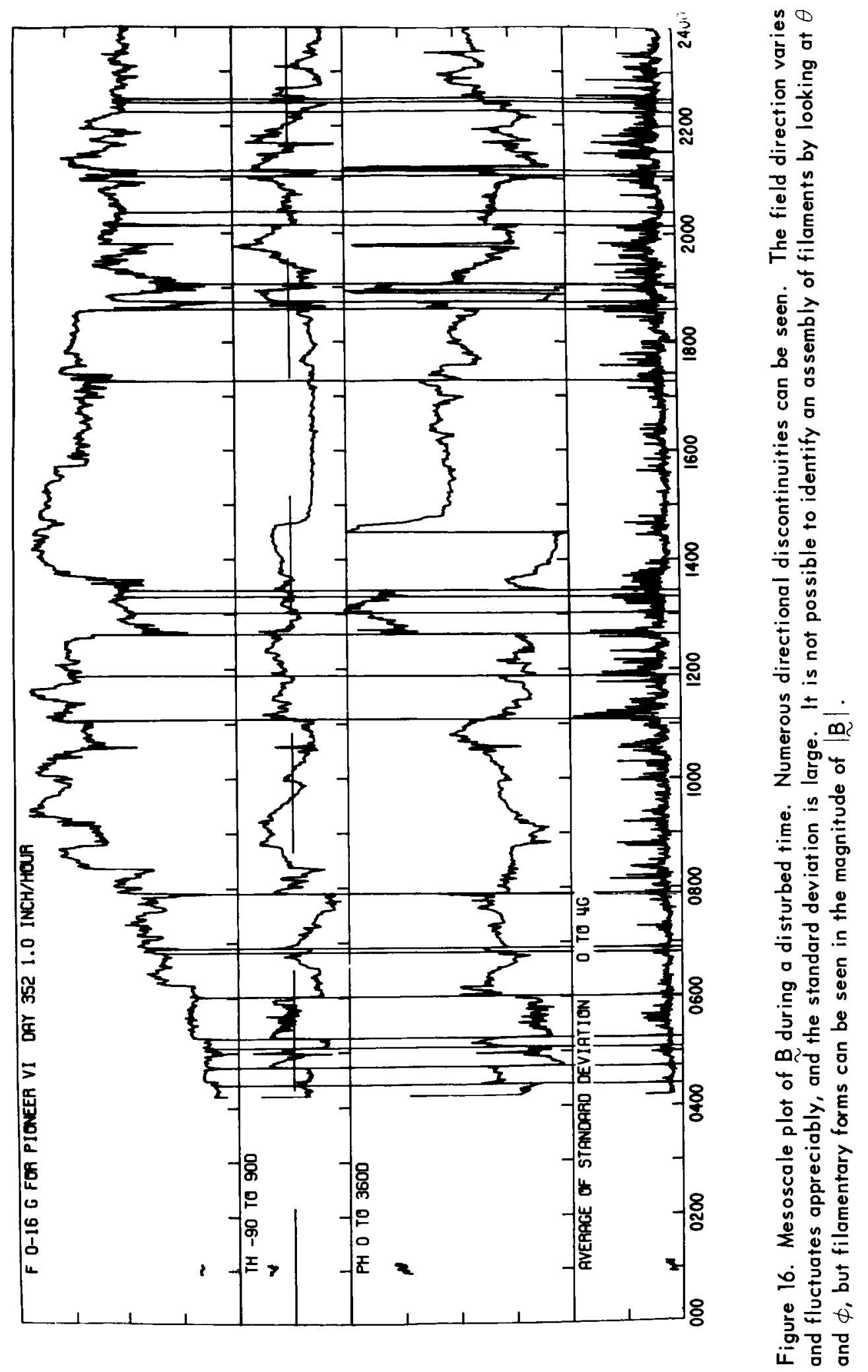

